\title{
Eigenvalue distributions for some correlated complex sample covariance matrices
}

\author{
P.J. Forrester \\ Department of Mathematics and Statistics, \\ University of Melbourne, Victoria 3010, Australia
}

\begin{abstract}
The distributions of the smallest and largest eigenvalues for the matrix product $Z^{\dagger} Z$, where $Z$ is an $n \times m$ complex Gaussian matrix with correlations both along rows and down columns, are expressed as $m \times m$ determinants. In the case of correlation along rows, these expressions are computationally more efficient than those involving sums over partitions and Schur polynomials reported recently for the same distributions.
\end{abstract}

\section{Introduction}

A typical setting in multivariate statistics is to measure each of $m$ variables $x_{1}, \ldots, x_{m}$ a total of $N$ times. For example, the variable $x_{k}$ may denote the wind speed at weather station $k$ at a specific time of day; recording the value on successive days gives a sequence of values $x_{k}^{(j)}, j=1, \ldots, N$ for the variable $x_{k}$, which forms a column vector $\vec{x}_{k}=\left[x_{k}^{(j)}\right]_{j=1, \ldots, N}$. Collecting together the column vectors for each of the variables $x_{k}$ gives the data matrix $X=\left[\vec{x}_{k}\right]_{k=1, \ldots, m}$. Let the average of the readings of variable $x_{k}$ be denoted $\bar{x}_{k}$, so that

$$
\bar{x}_{k}=\frac{1}{N} \sum_{j=1}^{N} x_{j} .
$$

Let $\vec{x}_{k}=\left[\bar{x}_{k}\right]_{j=1, \ldots, N}$ be the corresponding (constant) column vector, and set $\bar{X}:=\left[\vec{x}_{k}\right]_{k=1, \ldots, m}$. Forming now

$$
\frac{1}{n} A:=\frac{1}{n}(X-\bar{X})^{T}(X-\bar{X})=\left[\frac{1}{n} \sum_{j=1}^{N}\left(x_{k_{1}}^{(j)}-\bar{x}_{k_{1}}^{(j)}\right)\left(x_{k_{2}}^{(j)}-\bar{x}_{k_{2}}^{(j)}\right)\right]_{k_{1}, k_{2}=1, \ldots, m},
$$

$n=N-1$, gives an empirical approximation to the covariance matrix $\left[\left\langle\left(x_{k_{1}}-\left\langle x_{k_{1}}\right\rangle\right)\left(x_{k_{2}}-\left\langle x_{k_{2}}\right\rangle\right)\right]_{k_{1}, k_{2}=1, \ldots, m}\right.$ for the variables $\left\{x_{k}\right\}_{k=1, \ldots, m}$.

Analytic studies of the matrix $A$ can be carried out in the case that the variables $x_{1}, \ldots, x_{m}$ relating to the data matrix $X$ are chosen from a multivariate Gaussian distribution with variance matrix $\Sigma$ and mean $\vec{\mu}$. Then it is known (see e.g. 9]) that the distribution of $A$ is the same as that for the matrix product $Y^{T} Y$, where $Y$ is an $m \times n, n=N-1$, Gaussian matrix in which each row is drawn from a multivariate Gaussian distribution with covariance matrix $\Sigma$ and mean zero. Thus the joint probability density function (p.d.f.) of the elements of $Y$ is

$$
\frac{1}{C} e^{-\operatorname{Tr}\left(\Sigma^{-1} Y^{T} Y / 2\right)},
$$

where here and throughout (unless otherwise stated) $C$ represents some constant (i.e. quantity independent of the main variables of the equation, which here are the elements of $Y$ ). 
A standard practice in studying the empirical covariance matrix is to form the eigenvalue-eigenvector decomposition. This comes under the name of principal component analysis (see e.g. [11]). On a theoretical front one seeks analytic forms for eigenvalue distributions of the matrix $A=Y^{T} Y$ when $Y$ is distributed according to (1.1). In fact the eigenvalue p.d.f. can be written down in terms of a multivariable generalized hypergeometric function based on zonal polynomials (see e.g. [13]). This function is inherently difficult to compute, but there have been some recent advances [12. It is also possible to integrate over this p.d.f. to express the distribution of the largest eigenvalue as another generalized hypergeometric function [10].

In a recent work [15] a study of the p.d.f. for the smallest and largest eigenvalues of the matrix $A=Z^{\dagger} Z$ for $Z$ an $n \times m,(n \geq m)$ complex Gaussian matrix with p.d.f.

$$
\frac{1}{C} e^{-\operatorname{Tr}\left(\Sigma^{-1} Z^{\dagger} Z\right)}
$$

has been undertaken. Earlier studies had considered these p.d.f.'s in the case $\Sigma=I$ [ [] []. The setting of complex data matrices is of great importance in recent quantitative studies of wireless communication (see e.g. [18 17]). A significant feature of the p.d.f. (1.2) is that the corresponding joint eigenvalue p.d.f. of $A$ can be written as a determinant [8, 1, 2, 17. Moreover, as to be shown in Section 2 below, the marginal distributions by way of the p.d.f. of the smallest and largest eigenvalues can also be evaluated as determinants. In contrast, these same distributions where evaluated in [15] as a sum over Schur polynomials and as a generalized hypergeometric function based on the Schur polynomials respectively (see (2.15) and (2.9) below).

Suppose more generally that the complex data matrix $Z$ has p.d.f.

$$
\frac{1}{C} e^{-\operatorname{Tr}\left(\Sigma_{1}^{-1} Z^{\dagger} \Sigma_{2}^{-1} Z\right)}
$$

Here $\Sigma_{2}$ can be interpreted as the covariance coupling the measurements of a single variable $z_{k}$. Very recently [16, 17, it has been shown that for this distribution the canonical average

$$
\left\langle\operatorname{det}\left(1+u Z^{\dagger} Z\right)^{p}\right\rangle
$$

can be expressed as an $n \times n$ determinant, even though the joint eigenvalue p.d.f. of (1.2) cannot itself be written in a determinant form. In Section 3 we will use the method of [17] to similarly express the p.d.f. for the smallest and largest eigenvalues of $Z^{\dagger} Z$ with $Z$ distibuted as (1.3) in terms of determinants.

\section{Case of a single covariance matrix}

\subsection{Correlation across rows of $Z$}

Consider the p.d.f. (1.2). Introduce the singular value decomposition

$$
Z=U \operatorname{diag}\left(\mu_{1}, \ldots, \mu_{m}\right) V
$$

where $U(V)$ is a $m \times m(n \times n)$ unitary matrix and the $\mu_{j}^{2}=: \lambda_{j}$ are the eigenvalues of the positive definite matrix $Z^{\dagger} Z$.

We seek the joint distribution of the $\left\{\lambda_{j}\right\}_{j=1, \ldots, m}, p\left(\lambda_{1}, \ldots, \lambda_{m}\right)$ say. Firstly, with $A=Z^{\dagger} Z$, we know 
(see e.g. [5])

$$
\begin{aligned}
(d A) & =\frac{1}{C} \operatorname{det}\left(Z^{\dagger} Z\right)^{n-m}(d Z) \\
& =\frac{1}{C} \prod_{j=1}^{m} \lambda_{j}^{n-m} \prod_{1 \leq j<k \leq m}\left(\lambda_{k}-\lambda_{j}\right)^{2} d \lambda_{1} \cdots d \lambda_{m}\left(V^{\dagger} d V\right),
\end{aligned}
$$

where $\left(V^{\dagger} d V\right)$ is the Haar measure (uniform distribution) on the space of $m \times m$ unitary matrices $U(m)$. Thus

$$
p\left(\lambda_{1}, \ldots, \lambda_{m}\right)=\frac{1}{C} \prod_{j=1}^{m} \lambda_{j}^{n-m} \prod_{1 \leq j<k \leq m}\left(\lambda_{k}-\lambda_{j}\right)^{2} \int_{V \in U(m)} e^{-\operatorname{Tr}\left(\Sigma^{-1} V^{\dagger} \operatorname{diag}\left(\lambda_{1}, \ldots, \lambda_{m}\right) V\right)}\left(V^{\dagger} d V\right) .
$$

This is the well known Harish-Chandra/Itzykson-Zuber matrix integral (see e.g. 14]). It has a closed form determinantal evaluation, which when substituted in (2.3) implies

$$
p\left(\lambda_{1}, \ldots, \lambda_{m}\right)=\frac{1}{C} \prod_{j=1}^{m} \lambda_{j}^{n-m} \prod_{1 \leq j<k \leq m} \frac{\left(\lambda_{k}-\lambda_{j}\right)}{\left(s_{k}-s_{j}\right)} \operatorname{det}\left[e^{-s_{j} \lambda_{k}}\right]_{j, k=1, \ldots, m}
$$

where $\left\{s_{1}, \ldots, s_{m}\right\}$ are the eigenvalues of $\Sigma^{-1}$. As referenced in the third sentence below (1.2), the result (2.4) has been made explicit in a number of recent works.

Consider now the probability $E((\lambda, \infty))$ that the interval $(\lambda, \infty)$ is free of eigenvalues. This is related to the p.d.f. of the largest eigenvalue, $p^{\max }(\lambda)$ say, by

$$
p^{\max }(\lambda)=-\frac{d}{d \lambda} E((\lambda, \infty)) .
$$

We have

$$
\begin{aligned}
E((\lambda, \infty)) & :=\int_{0}^{\lambda} d \lambda_{1} \cdots \int_{0}^{\lambda} d \lambda_{m} p\left(\lambda_{1}, \ldots, \lambda_{m}\right) \\
& =\frac{1}{C} \frac{1}{\prod_{j<k}^{m}\left(s_{k}-s_{j}\right)} \int_{0}^{\lambda} d \lambda_{1} \cdots \int_{0}^{\lambda} d \lambda_{m} \prod_{j=1}^{m} \lambda_{j}^{n-m} \prod_{j<k}^{m}\left(\lambda_{k}-\lambda_{j}\right) \operatorname{det}\left[e^{-s_{j} \lambda_{k}}\right]_{j, k=1, \ldots, m} .
\end{aligned}
$$

Because both factors in the integrand are anti-symmetric in $\left\{\lambda_{j}\right\}_{j=1, \ldots, m}$, and

$$
\prod_{j<k}^{m}\left(\lambda_{k}-\lambda_{j}\right)=\operatorname{Asym} \lambda_{1}^{0} \lambda_{2} \cdots \lambda_{m}^{m-1},
$$

the product can be replaced by $\lambda_{1}^{0} \lambda_{2} \cdots \lambda_{m}^{m-1}$ provided we multiply by $m !$. Doing this we see the integrations over $\left\{\lambda_{k}\right\}$ can be performed column by column, to give

$$
E((\lambda, \infty))=\frac{m !}{C} \frac{1}{\prod_{j<k}^{m}\left(s_{k}-s_{j}\right)} \operatorname{det}\left[\int_{0}^{\lambda} t^{n-m+k-1} e^{-s_{j} t} d t\right]_{j, k=1, \ldots, m} .
$$

To evaluate $C$, we note $\lim _{\lambda \rightarrow \infty} E((\lambda, \infty))=1$. The integral in (2.5) can be evaluated in this limit to give

$$
1=\frac{m !}{C} \frac{1}{\prod_{j<k}^{m}\left(s_{k}-s_{j}\right)} \operatorname{det}\left[s_{j}^{-(n-m+k)}(n-m+k-1) !\right]_{j, k=1, \ldots, m} .
$$

Factoring the factorials from the determinant and then making use of the Vandermonde determinant formula shows

$$
C=(-1)^{m(m-1) / 2} m ! \prod_{k=1}^{m}(n-m+k-1) ! \prod_{j=1}^{m} s_{j}^{-n} .
$$


Substituting this in (2.5), and changing variables $s_{j} \mapsto \lambda s_{j}$ in the integral therein, we obtain for our final expression

$$
E((\lambda, \infty))=\frac{1}{\prod_{k=1}^{m}(n-m+k-1) !} \frac{\prod_{j=1}^{m}\left(\lambda s_{j}\right)^{n}}{\prod_{j<k}^{m}(-\lambda)\left(s_{k}-s_{j}\right)} \operatorname{det}\left[\int_{0}^{1} t^{n-m+k-1} e^{-\lambda s_{j} t} d t\right]_{j, k=1, \ldots, m} .
$$

We remark that in the case that $s_{j}=1(j=r+1, \ldots, m)$, the $m \rightarrow \infty$ limit of $E((\lambda, \infty))$, with $\lambda, s_{1}, \ldots, s_{r}$ appropriately scaled, is studied in [1]. We remark too that in [15, Corollary 3.3] $E((\lambda, \infty))$ is expressed in terms of the generalized multi-variable hypergeometric function

$$
{ }_{1} F_{1}\left(a, b ; x_{1}, \ldots, x_{m}\right):=\sum_{\kappa} \frac{[a]_{\kappa}}{d_{\kappa}^{\prime}[b]_{\kappa}} s_{\kappa}\left(x_{1}, \ldots, x_{m}\right) .
$$

In (2.8) $s_{\kappa}$ denotes the Schur polynomial labelled by a partition $\kappa=\left(\kappa_{1}, \ldots, \kappa_{m}\right), \kappa_{1} \geq \cdots \geq \kappa_{m}$,

$$
[a]_{\kappa}:=\prod_{j=1}^{m} \frac{\Gamma\left(a-j+1+\kappa_{j}\right)}{\Gamma(a-j+1)}
$$

while

$$
d_{\kappa}^{\prime}=\frac{[m]_{\kappa}}{\bar{f}_{m}(\kappa)}, \quad \bar{f}_{m}(\kappa):=\prod_{1 \leq i<j \leq m} \frac{\left(j-i+\kappa_{i}-\kappa_{j}\right)}{j-i} .
$$

Thus from [15] Eq. (3.5)]

$$
E((\lambda, \infty))=\prod_{k=1}^{m} \frac{\Gamma(k)}{\Gamma(n+k)} \prod_{j=1}^{m}\left(\lambda s_{j}\right)^{n}{ }_{1} F_{1}\left(n ; n+m ;-\lambda s_{1}, \ldots,-\lambda s_{m}\right) .
$$

Comparing (2.9) and (2.5) gives the determinant formula

$$
F_{1}\left(n ; n+m ; x_{1}, \ldots, x_{m}\right)=\prod_{k=1}^{m} \frac{\Gamma(n+k)}{\Gamma(k) \Gamma(n-m+k)} \frac{1}{\prod_{j<k}^{m}\left(x_{k}-x_{j}\right)} \operatorname{det}\left[\int_{0}^{1} t^{n-m+k-1} e^{x_{j} t} d t\right]_{j, k=1, \ldots, m} .
$$

The integral in (2.7) is itself a special case of a one variable confluent hypergeometric function ${ }_{1} F_{1}$, allowing us to write

$$
\begin{aligned}
E((\lambda, \infty))= & \frac{1}{\prod_{k=1}^{m}(n-m+k) !} \frac{\prod_{j=1}^{m}\left(\lambda s_{j}\right)^{n}}{\prod_{j<k}^{m}(-\lambda)\left(s_{k}-s_{j}\right)} \\
& \quad \times \operatorname{det}\left[{ }_{1} F_{1}\left(n-m+k ; n-m+k+1 ;-\lambda s_{j}\right)\right]_{j, k=1, \ldots, m} .
\end{aligned}
$$

Note that (2.11) and (2.9) are identical in the case $m=1$.

We draw attention to a limiting feature of (2.5) which is of relevance in the study of $E((\lambda, \infty))$ for fully correlated matrices (1.3). Suppose then that $n=m$ in (2.5), and consider the limit $s_{n} \rightarrow \infty$. Integrating the final row of integrals by parts, we see that the dominant term is that in the first column. Expanding by this term shows

$$
\left.\lim _{s_{n} \rightarrow \infty} E((\lambda, \infty))\right|_{m=n}=\left.E((\lambda, \infty))\right|_{m=n-1},
$$

and iterating this we have

$$
\left.\lim _{s_{n-m}, \ldots, s_{n} \rightarrow \infty} E((\lambda, \infty))\right|_{m=n}=E((\lambda, \infty))
$$

where on the right hand side $E((\lambda, \infty))$ is for the general $m$ case, as given by (2.7). The understanding of this result is that with $s_{n-m}, \ldots, s_{n} \rightarrow \infty$, the final $n-m$ rows of $Z$ become zero and so the eigenvalues of $Z^{\dagger} Z$ are those of $W^{\dagger} W$ for $W$ the restriction of $Z$ to its first $m$ rows, together with $m$ zero eigenvalues. 
For the probability $E((0, \lambda))$ that the interval $(0, \lambda)$ is free of eigenvalues, related to the p.d.f. of the smallest eigenvalue, $p^{\min }(\lambda)$ say, by

$$
p^{\min }(\lambda)=\frac{d}{d \lambda} E((0, \lambda))
$$

we have

$$
\begin{aligned}
E((0, \lambda)) & :=\int_{\lambda}^{\infty} d \lambda_{1} \cdots \int_{\lambda}^{\infty} d \lambda_{m} p\left(\lambda_{1}, \ldots, \lambda_{m}\right) \\
& =\frac{1}{C} \frac{1}{\prod_{j<k}\left(s_{k}-s_{j}\right)} \int_{\lambda}^{\infty} d \lambda_{1} \cdots d \lambda_{m} \prod_{j=1}^{m} \lambda_{j}^{n-m} \prod_{1 \leq j<k \leq m}\left(\lambda_{k}-\lambda_{j}\right) \operatorname{det}\left[e^{-s_{j} \lambda_{k}}\right]_{j, k=1, \ldots, m} .
\end{aligned}
$$

Proceeding now as in the derivation of (2.7) shows

$$
\begin{aligned}
E((0, \lambda)) & =\frac{m !}{C} \frac{1}{\prod_{j<k}^{m}\left(s_{k}-s_{j}\right)} \operatorname{det}\left[\int_{\lambda}^{\infty} t^{n-m+k-1} e^{-s_{j} t} d t\right]_{j, k=1, \ldots, m} \\
& =\frac{m !}{C} \frac{e^{-\lambda \sum_{j=1}^{m} s_{j}}}{\prod_{j<k}^{m}\left(s_{k}-s_{j}\right)} \operatorname{det}\left[\int_{0}^{\infty}(t+\lambda)^{n-m+k-1} e^{-s_{j} t} d t\right]_{j, k=1, \ldots, m}
\end{aligned}
$$

where $C$ is given by (2.6). Note that for $m=n$ the determinant only contributes a constant (i.e. term independent of $\lambda$ ) and we have

$$
\left.E((0, \lambda))\right|_{m=n}=e^{-\lambda \sum_{j=1}^{n} s_{j}}
$$

which generalizes the same result known for $s_{1}=\cdots=s_{n}=1$ [4] [6].

In terms of the confluent hypergeometric function

$$
U(a, b, z):=\frac{1}{\Gamma(a)} \int_{0}^{\infty} e^{-z t} t^{a-1}(1+t)^{b-a-1} d t,
$$

which unlike ${ }_{1} F_{1}(a, b ; z)$ is singular at $z=0$, but like ${ }_{1} F_{1}$ is available as an inbuilt function on a number of mathematical computing packages, (2.12) reads

$$
\begin{aligned}
E((0, \lambda))= & (-1)^{m(m-1) / 2} \prod_{k=1}^{m} \frac{\left(\lambda s_{k}\right)^{n}}{\Gamma(n-m+k)} \frac{e^{-\lambda \sum_{j=1}^{m} s_{j}}}{\prod_{j<k}^{m}\left(s_{k}-s_{j}\right)} \\
& \times \operatorname{det}\left[U\left(1, n-m+k+1, \lambda s_{j}\right)\right]_{j, k=1, \ldots, m} .
\end{aligned}
$$

The computationally more complex evaluation

$$
E((0, \lambda))=e^{-\lambda \sum_{j=1}^{m} s_{j}} \sum_{k=0}^{m(n-m)} \lambda^{k} \sum_{\substack{\kappa:|\kappa|=k \\ \kappa_{1} \leq n-m}} \frac{s_{\kappa}\left(s_{1}, \ldots, s_{m}\right)}{d_{\kappa}^{\prime}}
$$

is given in [15, Eq. (3.8)].

Also given in [15] is an expression involving sums over partitions and Schur polynomials for the probability $E((0, a) \cup(b, \infty))$ that there are no eigenvalues in either of the intervals $(0, a)$ or $(b, \infty)$. In terms of this quantity the joint p.d.f. for the smallest and largest eigenvalues, $p(a, b)$ say, is given by

$$
p(a, b)=-\frac{\partial^{2}}{\partial a \partial b} E((0, a) \cup(b, \infty)) .
$$

From $p(a, b)$ one can deduce the distribution of $b / a$, which is the square of the condition number and so 
is of relevance in numerical analysis. The method of derivation of (2.7) yields the determinant evaluation

$$
\begin{aligned}
E & ((0, a) \cup(b, \infty)) \\
& =\frac{1}{C} \frac{1}{\prod_{j<k}^{m}\left(s_{k}-s_{j}\right)} \int_{a}^{b} d \lambda_{1} \cdots \int_{a}^{b} d \lambda_{m} \prod_{j=1}^{m} \lambda_{j}^{n-m} \prod_{j<k}^{m}\left(\lambda_{k}-\lambda_{j}\right) \operatorname{det}\left[e^{-s_{j} \lambda_{k}}\right]_{j, k=1, \ldots, m} \\
& =\frac{m !}{C} \frac{1}{\prod_{j<k}^{m}\left(s_{k}-s_{j}\right)} \operatorname{det}\left[\int_{a}^{b} t^{n-m+k-1} e^{-s_{j} t} d t\right]_{j, k=1, \ldots, m}
\end{aligned}
$$

where $C$ is given by (2.6).

\subsection{Correlation down columns of $Z$}

Consider next the case that $Z$ has distribution

$$
\frac{1}{C} e^{-\operatorname{Tr}\left(Z^{\dagger} \Sigma_{2}^{-1} Z\right)}
$$

(1.3) with $\Sigma_{1}=I$ ). Now

$$
e^{-\operatorname{Tr}\left(Z^{\dagger} \Sigma_{2}^{-1} Z\right)}=e^{-\operatorname{Tr}\left(\Sigma_{2}^{-1} Z Z^{\dagger}\right)}
$$

and the non-zero eigenvalues of $Z Z^{\dagger}$ agree with the eigenvalues of $Z^{\dagger} Z$, which we again denote $\left\{\lambda_{j}\right\}_{j=1, \ldots, m}$. With $A=Z Z^{\dagger}$ (2.2) again applies but with $V$ replaced by $U$ (recall (2.1)), and thus

$$
\begin{aligned}
& p\left(\lambda_{1}, \ldots, \lambda_{m}\right) \\
& \quad=\frac{1}{C} \prod_{j=1}^{m} \lambda_{j}^{n-m} \prod_{1 \leq j<k \leq m}\left(\lambda_{k}-\lambda_{j}\right)^{2} \lim _{\lambda_{m+1}, \ldots, \lambda_{n} \rightarrow 0} \int_{U \in U(n)} e^{-\operatorname{Tr}\left(\Sigma_{2}^{-1} U \operatorname{diag}\left(\lambda_{1}, \ldots, \lambda_{n}\right) U^{\dagger}\right)}\left(U^{\dagger} d U\right) .
\end{aligned}
$$

(Here the limit could have been taken immediately, but as noted in [17 there are computational advantages in delaying this step.) Proceeding as in the derivation of (2.4) gives

$$
p\left(\lambda_{1}, \ldots, \lambda_{m}\right)=\frac{1}{C} \lim _{\lambda_{m+1}, \ldots, \lambda_{n} \rightarrow 0} \frac{\prod_{j=1}^{m} \lambda_{j}^{n-m} \prod_{j<k}^{m}\left(\lambda_{k}-\lambda_{j}\right)^{2}}{\prod_{j<k}^{n}\left(\lambda_{k}-\lambda_{j}\right)\left(s_{k}-s_{j}\right)} \operatorname{det}\left[e^{-s_{j} \lambda_{k}}\right]_{j, k=1, \ldots, n}
$$

where here $\left\{s_{1}, \ldots, s_{n}\right\}$ denotes the eigenvalues of $\Sigma_{2}^{-1}$. Now taking the limit this reads

$$
p\left(\lambda_{1}, \ldots, \lambda_{m}\right)=\frac{1}{C} \frac{\prod_{j<k}^{m}\left(\lambda_{k}-\lambda_{j}\right)}{\prod_{j<k}^{n}\left(s_{k}-s_{j}\right)} \operatorname{det}\left[\left[e^{-s_{j} \lambda_{k}}\right]_{\substack{j=1, \ldots, n \\ k=1, \ldots, m}}\left[s_{j}^{k-1}\right]_{\substack{j=1, \ldots, n \\ k=1, \ldots, n-m}}\right] .
$$

The joint p.d.f. 2.20) has been derived previously in [3] 17.

The derivations of (2.7) and (2.12) can be applied to (2.20) to deduce determinant formulas for $E((\lambda, \infty))$ and $E((0, \lambda))$. Thus we find

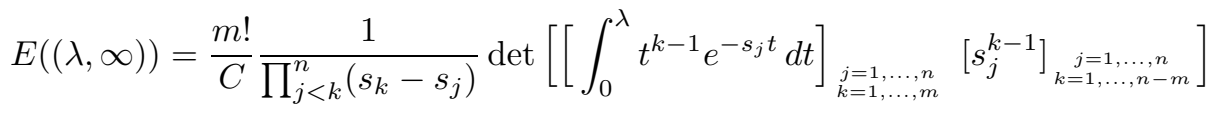

and

$$
E((0, \lambda))=\frac{\prod_{k=1}^{m} k !}{C} \frac{e^{-\lambda \sum_{j=1}^{n} s_{j}}}{\prod_{j<k}^{n}\left(s_{k}-s_{j}\right)} \operatorname{det}\left[\left[s_{j}^{-k}\right]_{\substack{j=1, \ldots, n \\ k=1, \ldots, m}}\left[e^{\lambda s_{j}} s_{j}^{k-1}\right]_{\substack{j=1, \ldots, n \\ k=1, \ldots, n-m}}\right]
$$

where

$$
C=\prod_{k=1}^{m} k ! \prod_{j=1}^{n} s_{j}^{-m}
$$




\section{Fully correlated case}

We turn our attention now to the case (1.3), in which the data matrix $Z$ is correlated both across rows and down columns. Here it does not appear possible to write the joint eigenvalue p.d.f. of $Z^{\dagger} Z$ in determinant form. Nonetheless, it has been shown recently by Simon and Moustakos [16 (see [17 for a detailed presentation) that it is possible to give a determinant formula for the average (1.4). Here we will show that their calculation can be adopted to give determinant formulas for $E((\lambda, \infty))$ and $E((0, \lambda))$ (the latter being restricted to the case $m=n$ ).

To begin we suppose $m=n$. In the case of $E((\lambda, \infty))$, by using the limiting procedure discussed in the paragraph below (2.10), a formula can be deduced from this for general $m \leq n$. Our starting point is the formula 17 .

$$
p\left(\lambda_{1}, \ldots, \lambda_{n}\right)=\prod_{j=1}^{n}\left(r_{j} s_{j}\right)^{n} \prod_{j<k}^{n}\left(\lambda_{k}-\lambda_{j}\right) \mathcal{I}\left(\left\{r_{i}\right\},\left\{s_{i}\right\},\left\{\lambda_{i}\right\}\right)
$$

where, with the eigenvalues of $\Sigma_{1}^{-1}, \Sigma_{2}^{-1}$ given by $\left\{r_{i}\right\},\left\{s_{i}\right\}$ respectively,

$$
\begin{aligned}
& \mathcal{I}\left(\left\{r_{i}\right\},\left\{s_{i}\right\},\left\{\lambda_{i}\right\}\right) \\
& \quad=\frac{1}{n !} \sum_{k_{1}>k_{2}>\cdots>k_{n} \geq 0} \prod_{j=1}^{n} \frac{(-1)^{k_{j}}}{k_{j} !} \frac{\operatorname{det}\left[r_{j}^{k_{l}}\right] \operatorname{det}\left[\lambda_{j}^{k_{l}}\right] \operatorname{det}\left[s_{j}^{k_{l}}\right]}{\prod_{j<l}^{n}\left(k_{l}-k_{j}\right)\left(r_{l}-r_{j}\right)\left(s_{l}-s_{j}\right)} .
\end{aligned}
$$

In [17, Lemma 5] it is proved that $\mathcal{I}$ is bounded by an exponentially decaying function in $\lambda_{j}$ for each $j=1, \ldots, n$.

Consider first $E((0, \lambda))$. We thus seek to integrate each $\lambda_{i}$ in (3.1) over $(\lambda, \infty)$. For this we take inspiration from [17] and note from the Vandermonde determinant evaluation that

$$
\prod_{j<k}^{n}\left(\lambda_{k}-\lambda_{j}\right)=\prod_{j=1}^{n}\left(\frac{\lambda_{j}-\lambda}{\lambda^{1 / 2}}\right)^{n-1} \operatorname{det}\left[\left(\frac{\lambda_{j}}{\lambda_{j}-\lambda}\right)^{k-1}\right]_{j, k=1, \ldots, n} .
$$

Expanding out the determinant according to its definition, substituting in (3.1) and integrating gives

$$
\begin{aligned}
\left.E((0, \lambda))\right|_{m=n}= & \prod_{j=1}^{n}\left(r_{j} s_{j}\right)^{n} \lambda^{-n(n-1) / 2} \sum_{P \in S_{N}} \varepsilon(P) \int_{\lambda}^{\infty} d \lambda_{1} \cdots \int_{\lambda}^{\infty} d \lambda_{N} \\
& \times \prod_{j=1}^{n}\left(\lambda_{j}-\lambda\right)^{n-P(j)} \lambda_{j}^{P(j)-1} \mathcal{I}\left(\left\{r_{i}\right\},\left\{s_{i}\right\},\left\{\lambda_{i}\right\}\right) .
\end{aligned}
$$

For each integration variable, we integrate by parts $P(j)-1$ times, making use of the simple formula

$$
\left(\lambda_{j}-\lambda\right)^{m}=\frac{1}{m+1} \frac{\partial}{\partial \lambda_{j}}\left(\lambda_{j}-\lambda\right)^{m+1}, \quad m \neq-1 .
$$

For $m>-1,\left(\lambda_{j}-\lambda\right)^{m+1}$ vanishes at $\lambda_{j}=\lambda$ (this is part of the motivation for the manipulation (3.3)), while from the remark below (3.2) the factor involving $\mathcal{I}$ vanishes at $\lambda_{j}=\infty$. Hence in the integration by parts there is no contribution from the end points. We must compute the partial derivatives with

respect to $\lambda_{j}$ of $\lambda_{j}^{P(j)-1} \mathcal{I}$. For this note that the only term dependent of $\lambda_{j}$ in $\mathcal{I}$ is $\operatorname{det}\left[\lambda_{j}^{k_{l}}\right]$, and

$$
\prod_{j=1}^{n} \lambda_{j}^{P(j)-1} \operatorname{det}\left[\lambda_{j}^{k_{l}}\right]=\operatorname{det}\left[\lambda_{j}^{k_{l}+P(j)-1}\right]
$$


Performing $P(j)-1$ integration by parts in each variable $\lambda_{j}$ thus gives

$$
\begin{aligned}
\left.E((0, \lambda))\right|_{m=n}= & \prod_{j=1}^{n}\left(r_{j} s_{j}\right)^{n} \frac{\lambda^{-n(n-1) / 2}}{n !} \sum_{P \in S_{N}} \varepsilon(P) \int_{\lambda}^{\infty} d \lambda_{1} \cdots \int_{\lambda}^{\infty} d \lambda_{N} \\
& \times \prod_{j=1}^{n}\left(\lambda_{j}-\lambda\right)^{(n-1)} \sum_{k_{1}>\cdots>k_{n} \geq 0} \prod_{j=1}^{n} \frac{(-1)^{k_{j}}}{k_{j} !} \\
& \times \frac{\operatorname{det}\left[r_{j}^{k_{l}}\right] \operatorname{det}\left[\prod_{p=1}^{P(j)-1}\left(-\frac{k_{l}+p}{n-p}\right) \lambda_{j}^{k_{l}}\right] \operatorname{det}\left[s_{j}^{k_{l}}\right]}{\prod_{j<l}^{n}\left(k_{l}-k_{j}\right)\left(r_{l}-r_{j}\right)\left(s_{l}-s_{j}\right)} .
\end{aligned}
$$

Next we want to integrate row by row in the determinant. Although the integrand decays exponentially at infinity, this gives divergent integrals, as a result of interchanging the order of summation and integration. To overcome this, write

$$
\int_{\lambda}^{\infty} d \lambda_{j}=\lim _{L \rightarrow \infty} \int_{\lambda}^{L} d \lambda_{j} \quad(j=1, \ldots, n)
$$

and so interchange only the finite range integrals with the summation of $\left\{h_{j}\right\}$. We see the resulting one dimensional integrals are the same down each column of the determinant and so can be factored. Furthermore, the sum over $P \in S_{N}$ then simply interchanges rows in the determinant, which is compenstated for by $\varepsilon(P)$, thus contributing an overall factor of $n$ !. Hence

$$
\begin{gathered}
\left.E((0, \lambda))\right|_{m=n}=\prod_{j=1}^{n}\left(r_{j} s_{j}\right)^{n} \lambda^{-n(n-1) / 2} \lim _{L \rightarrow \infty} \sum_{k_{1}>\cdots>k_{n} \geq 0} \prod_{j=1}^{n} \frac{(-1)^{k_{j}}}{k_{j} !} \frac{\operatorname{det}\left[r_{j}^{k_{l}}\right] \operatorname{det}\left[s_{j}^{k_{l}}\right]}{\prod_{j<l}^{n}\left(k_{l}-k_{j}\right)\left(r_{l}-r_{j}\right)\left(s_{l}-s_{j}\right)} \\
\prod_{l=1}^{n} \int_{\lambda}^{L}(t-\lambda)^{n-1} t^{k_{l}} d t \operatorname{det}\left[\prod_{p=1}^{j-1}\left(-\frac{k_{l}+p}{n-p}\right)\right]_{j, l=1, \ldots, n} .
\end{gathered}
$$

As noted in [17, it is straightforward to verify that

$$
\operatorname{det}\left[\prod_{p=1}^{j-1}\left(-\frac{k_{l}+p}{n-p}\right)\right]_{j, l=1, \ldots, n}=(-1)^{n(n-1) / 2} \prod_{j=1}^{n-1} \frac{1}{j^{j}} \prod_{j<l}^{n}\left(k_{l}-k_{j}\right),
$$

thus cancelling $\prod_{j<l}^{n}\left(k_{l}-k_{j}\right)$ and reducing (3.6) to

$$
\begin{aligned}
\left.E((0, \lambda))\right|_{m=n}= & \prod_{j=1}^{n}\left(r_{j} s_{j}\right)^{n}(-\lambda)^{-n(n-1) / 2} \prod_{j=1}^{n-1} \frac{1}{j^{j}} \lim _{L \rightarrow \infty} \sum_{k_{1}>\cdots k_{n} \geq 0} \\
& \times \prod_{j=1}^{n}\left(\int_{\lambda}^{L}(t-\lambda)^{n-1} \frac{(-t)^{k_{j}}}{k_{j} !}\right) \frac{\operatorname{det}\left[r_{j}^{k_{l}}\right] \operatorname{det}\left[s_{j}^{k_{l}}\right]}{\prod_{j<l}^{n}\left(k_{l}-k_{j}\right)\left(r_{l}-r_{j}\right)\left(s_{l}-s_{j}\right)}
\end{aligned}
$$

The lattice version of the well known Heine formula from random matrix theory (see e.g. [5]),

$$
\begin{gathered}
\int_{I} d \mu\left(x_{1}\right) \cdots \int_{I} d \mu\left(x_{N}\right) \operatorname{det}\left[\phi_{j}\left(x_{k}\right)\right]_{j, k=1, \ldots, N} \operatorname{det}\left[\psi_{j}\left(x_{k}\right)\right]_{j, k=1, \ldots, N} \\
=N ! \operatorname{det}\left[\int_{I} \phi_{j}(x) \psi_{k}(x) d \mu(x)\right]_{j, k=1, \ldots, N}
\end{gathered}
$$

namely

$$
\sum_{k_{1}>\cdots>k_{n} \geq 0} \operatorname{det}\left[a_{i}^{k_{j}}\right] \operatorname{det}\left[b_{i}^{k_{j}}\right] \prod_{i=1}^{n} w\left(k_{i}\right)=\operatorname{det}\left[\sum_{p=0}^{\infty} w(p)\left(a_{i} b_{j}\right)^{p}\right]_{i, j=1, \ldots, n}
$$


referred to in [17] as the Cauchy-Binet formula, allows the sum in (3.7) to be computed. Taking then the limit $L \rightarrow \infty$ gives the sought determinant formula

$$
\begin{aligned}
\left.E((0, \lambda))\right|_{m=n}= & \prod_{j=1}^{n}\left(r_{j} s_{j}\right)^{n}(-\lambda)^{-n(n-1) / 2} \prod_{j=1}^{n-1} \frac{1}{j^{j}} \frac{1}{\prod_{j<l}^{n}\left(r_{l}-r_{j}\right)\left(s_{l}-s_{j}\right)} \\
& \times \operatorname{det}\left[\int_{\lambda}^{\infty}(t-\lambda)^{n-1} e^{-t r_{j} s_{l}} d t\right]_{j, l=1, \ldots, n}
\end{aligned}
$$

And changing variables $t \mapsto t+\lambda$ in the integral allows [3.8) to be simplified further, giving

$$
\left.E((0, \lambda))\right|_{m=n}=\prod_{j=1}^{n-1} j ! \frac{1}{\prod_{j<l}^{n}(-\lambda)\left(r_{l}-r_{j}\right)\left(s_{l}-s_{j}\right)} \operatorname{det}\left[e^{-\lambda r_{j} s_{l}}\right]_{j, l=1, \ldots, n} .
$$

Curiously this is the Harish-Chandra/Itzykson-Zuber matrix integral evaluation used in going from (2.3) to (2.4) and so we have the matrix integral representation

$$
\left.E((0, \lambda))\right|_{m=n}=\int e^{-\operatorname{Tr}\left(\lambda R V^{\dagger} S V\right)}\left[V^{\dagger} d V\right],
$$

where $R, S$ are Hermitian matrices has eigenvalues $\left\{r_{i}\right\},\left\{s_{i}\right\}$ respectively, and $\left[V^{\dagger} d V\right]$ denotes the normalized Haar measure, $\int\left[V^{\dagger} d V\right]=1$. With $m=n$, in the limit $s_{1}, \ldots, s_{n} \rightarrow 1$ the p.d.f. (1.3) reduces to (1.2). In keeping with this we can check that (3.9) reduces to (2.13) (with $s_{j} \mapsto r_{j}(j=1, \ldots, n)$ in the latter).

We turn our attention now to $E((\lambda, \infty)$. For this we use a minor rewrite of (3.3)

$$
\prod_{j<k}^{n}\left(\lambda_{k}-\lambda_{j}\right)=\prod_{j=1}^{n}\left(\frac{\lambda-\lambda_{j}}{\lambda^{1 / 2}}\right)^{n-1} \operatorname{det}\left[\left(\frac{\lambda_{j}}{\lambda-\lambda_{j}}\right)^{k-1}\right] .
$$

in (3.1) so that the analogue of (3.4) reads

$$
\begin{aligned}
\left.E((\lambda, \infty))\right|_{m=n}= & \prod_{j=1}^{n}\left(r_{j} s_{j}\right)^{n} \lambda^{-n(n-1) / 2} \sum_{P \in S_{N}} \varepsilon(P) \int_{0}^{\lambda} d \lambda_{1} \cdots \int_{0}^{\lambda} d \lambda_{N} \\
& \times \prod_{j=1}^{n}\left(\lambda-\lambda_{j}\right)^{n-P(j)} \lambda_{j}^{P(j)-1} \mathcal{I}\left(\left\{r_{i}\right\},\left\{s_{i}\right\},\left\{\lambda_{i}\right\}\right) .
\end{aligned}
$$

The procedure of going from (3.4) to (3.8) can now be enacted. (Note that in the integration by parts the factor

$$
\prod_{j=1}^{n}\left(\lambda-\lambda_{j}\right)^{n-P(j)} \lambda_{j}^{P(j)-1}
$$

ensures that the integrand vanishes at the end points.) We thus arrive at the determinant evaluation

$$
\left.E((\lambda, \infty))\right|_{m=n}=\prod_{j=1}^{n-1} \frac{1}{j^{j}} \frac{\prod_{j=1}^{n}\left(\lambda r_{j} s_{j}\right)^{n}}{\prod_{j<l}^{n}(-\lambda)\left(r_{l}-r_{j}\right)\left(s_{l}-s_{j}\right)} \operatorname{det}\left[\int_{0}^{1}(1-t)^{n-1} e^{-\lambda r_{j} s_{l} t} d t\right]_{j, l=1, \ldots, n} .
$$

Analogous to the remark in the paragraph below (3.10), we must have that for $s_{1}, \ldots, s_{n} \rightarrow 1$ (3.11) coincides with (2.7) (after setting $s_{j} \mapsto r_{j}(j=1, \ldots, n)$ in the latter). Now, taking the limit $s_{1}, \ldots, s_{n} \rightarrow$ 1 in (3.11) gives

$$
\left(\frac{1}{(n-1) !}\right)^{n-1} \frac{\prod_{j=1}^{n}\left(\lambda r_{j}\right)^{n}}{\prod_{j<l}(-\lambda)\left(r_{l}-r_{j}\right)} \operatorname{det}\left[\int_{0}^{1} e^{-\lambda r_{j} t} \frac{d^{k-1}}{d t^{k-1}}\left(t^{k-1}(1-t)^{n-1}\right) d t\right]_{j, k=1, \ldots, n}
$$


Expanding the derivatives using the product rule, we see after elementary column operations that the determinant in (3.12) is equal to

$$
\begin{aligned}
\operatorname{det} & {\left[\int_{0}^{1} e^{-\lambda r_{j} t} t^{k-1} \frac{d^{k-1}}{d t^{k-1}}\left(t^{k-1}(1-t)^{n-1}\right) d t\right]_{j, k=1, \ldots, n} } \\
& =\prod_{k=1}^{n-1} \frac{(n-1) !}{(n-k) !} \operatorname{det}\left[\int_{0}^{1} e^{-\lambda r_{j} t}(1-t)^{n-1}\left(\frac{t}{1-t}\right)^{k-1} d t\right]_{j, k=1, \ldots, n} .
\end{aligned}
$$

But the determinant in (3.13) can be written

$$
\begin{aligned}
& \int_{0}^{1} d t_{1} \cdots \int_{0}^{1} d t_{n} \prod_{j=1}^{n} e^{-\lambda r_{j} t_{j}}\left(1-t_{j}\right)^{n-1} \operatorname{det}\left[\left(\frac{t_{j}}{1-t_{j}}\right)^{k-1}\right]_{j, k=1, \ldots, n} \\
& =\int_{0}^{1} d t_{1} \cdots \int_{0}^{1} d t_{n} \prod_{j=1}^{n} e^{-\lambda r_{j} t_{j}} \prod_{j<k}\left(t_{k}-t_{j}\right) \operatorname{det}\left[\int_{0}^{1} e^{-\lambda r_{j} t} t^{k-1} d t\right]_{j, k=1, \ldots, n}
\end{aligned}
$$

Substituting (3.14) in (3.13), then substituting the result in (3.12) reclaims (2.7) in the case $m=n$.

It remains to apply the limiting procedure discussed in the paragraph below (2.10) to deduce from (3.11) the evaluation for general $m \leq n$. Noting the asymptotic expansion

$$
\int_{0}^{1}(1-t)^{n-1} e^{-\lambda r_{j} s_{l} t} d t \sim \sum_{p=1}^{n}(-1)^{p-1} \frac{(n-1) \cdots(n-p+1)}{\left(\lambda r_{j} s_{l}\right)^{p}}
$$

the required limits can be taken to give

$$
\begin{aligned}
E((\lambda, \infty))=(-1)^{(n-m)(n-m-1) / 2} \prod_{j=1}^{n-1} \frac{1}{j^{j}} \prod_{p=1}^{n-m-1} \frac{\Gamma(n)}{\Gamma(n-p)} \frac{\left(\prod_{j=1}^{m} r_{j}\right)^{n}\left(\prod_{j=1}^{n} \lambda s_{j}\right)^{n}}{\prod_{j<l}^{m}\left(r_{l}-r_{j}\right) \prod_{j<l}^{n} \lambda\left(s_{l}-s_{j}\right)} \\
\times \operatorname{det}\left[\begin{array}{c}
{\left[\int_{0}^{1}(1-t)^{n-1} e^{-\lambda r_{j} s_{l} t} d t\right]_{\substack{j=1, \ldots, m \\
l=1, \ldots, n}}} \\
\left.\left[\left(\lambda s_{l}\right)^{-j}\right] \begin{array}{c}
\substack{j=1, \ldots, n-m \\
l=1, \ldots, n} \\
l
\end{array}\right]
\end{array}\right.
\end{aligned}
$$

\section{Acknowledgement}

This work was supported by the Australian Research Council.

\section{References}

[1] J. Baik, G. Ben Arous, and S. Péché. Phase transition of the largest eigenvalue for nonnull complex sample covariance matrices. Annals of Prob., 33:1643-1697, 2005.

[2] P.M. Bleher and A. Kuijlaars. Integral representations for multiple Hermite and Laguerre polynomials. arXiv:math.CA/0406616

[3] M. Chiani, M.Z. Win, and A. Zanella. On the capacity of spatially correlated MIMO Rayleigh-fading channels. IEEE Trans. Inform. Theory, 49:2363-2371, 2003.

[4] A. Edelman. Eigenvalues and condition numbers of random matrices. SIAM J. Matrix Anal. Appl., 9:543-560, 1988. 
[5] P.J. Forrester. Log-gases and Random Matrices. www.ms.unimelb.edu.au/ ${ }^{\sim}$ matpjf/matpjf.html.

[6] P.J. Forrester. Exact results and universal asymptotics in the Laguerre random matrix ensemble. $J$. Math. Phys., 35:2539-2551, 1993.

[7] P.J. Forrester and T.D. Hughes. Complex Wishart matrices and conductance in mesoscopic systems: exact results. J. Math. Phys., 35:6736-6747, 1994.

[8] H. Gao and P.J. Smith. A determinant representation for the distribution of quadratic forms in complex normal vectors. J. Mult. Anal., 73:155-165, 2000.

[9] A.K. Gupta and D.K. Nagar. Matrix variate distributions. Chapman \& Hall/CRC, Boca Raton, 1999.

[10] A.T. James. Distributions of matrix variate and latent roots derived from normal samples. Ann. Math. Statist., 35:475-501, 1964.

[11] I.M. Johnstone. On the distribution of the largest principal component. Ann. Math. Stat., 29:295$327,2001$.

[12] P. Koev and A. Edelman. The efficient evaluation of the hypergeometric function of a matrix argument. arXiv:math.PR/0505344, 2005.

[13] R.J. Muirhead. Aspects of multivariable statistical theory. Wiley, New York, 1982.

[14] A.Y. Orlov. New solvable matrix integrals. Int. J. Mod. Phys. A, 19:276-293, 2004.

[15] T. Ratnarajah, R. Vaillancourt, and M. Alvo. Eigenvalues and condition numbers of complex random matrices. SIAM J. Matrix Anal. Appl., 26:441-456, 2005.

[16] S.H. Simon and A. L. Moustakas. Eigenvalue density of correlated random Wishart matrices. Phys. Rev. E, 69:065101(R), 2004.

[17] S.H. Simon, A. L. Moustakas, and L. Marinelli. Capacity and character expansions: moment generating function and other exact results for MIMO correlated channels. arXiv:cs.IT/0509080 2005.

[18] A.M. Tulino and S. Verdú. Random matrix theory and wireless communications. volume 1 of Foundations and Trends in Communcations and Information Theory, pages 1-182. Now, 2004. 